\title{
Enseñanza de medicina del trabajo en el pregrado de las escuelas de medicina en México
}

\author{
Francisco Raúl Sánchez-Román, MD, M en C,(I) Alda María Medina-Figueroa, MC,(I) \\ Ricardo Alfonso Rangel-Zertuche, MC,(2) Apolinar Sánchez-Ramos, MC.(3)
}

\section{Sánchez-Román FR, Medina-Figueroa AM, Rangel-Zertuche RA, Sánchez-Ramos A. Enseñanza de medicina del trabajo en el pregrado de las escuelas de medicina en México. Salud Publica Mex 2009;51:97-103.}

\section{Resumen}

Objetivo. Analizar el estado actual de la enseñanza de medicina del trabajo (MT) en facultades y escuelas de medicina en México. Material y métodos. Se llevó a cabo una encuesta descriptiva. Se identificaron facultades y escuelas a través de directorios de organizaciones de educación superior. Para el análisis de la información, se utilizó estadística descriptiva e inferencial. Resultados. De las 75 escuelas y facultades de medicina, 39 (52\%) ofrecen la asignatura como obligatoria, con predominio en planteles públicos $(p<0.02)$. Sólo 15 (38\%) cuentan con especialistas en MT como profesores. Conclusiones. La disparidad en la enseñanza de aspectos básicos sobre MT en el pregrado explica en parte el poco desarrollo y el pobre reconocimiento social y profesional de la especialidad, lo que desencadena problemas para la salud pública derivados de la escasa prevención de riesgos de trabajo y de conservación de la salud de los trabajadores en los centros laborales.

Palabras clave: medicina del trabajo; educación médica; educación de pregrado en medicina, México
Sánchez-Román FR, Medina-Figueroa AM,

Rangel-Zertuche RA, Sánchez-Ramos A.

The teaching of occupational medicine

in Mexican medical schools.

Salud Publica Mex 2009;5 I:97-103.

\section{Abstract}

Objective. To analyze the current situation of teaching occupational medicine (OM) in academic programs and medical schools in Mexico. Material and methods. A descriptive survey was conducted and schools were identified through the main directories of medical schools. For the analysis of information descriptive and inferential statistics were used. Results. A total of 75 medical schools were identified. In $39(52 \%)$ the subject is mandatory, with a predominance in public schools $(p<0.02)$. Among the schools that offer the subject, only $15(38 \%)$ have professors specialized in OM. Conclusions. Disparity in teaching basic aspects of OM in medical schools explains the little development and social and professional recognition of the specialty; it also highlights serious problems for public health, derived from the lack of prevention of risks in work environments.

Key words: occupational medicine; medical education; undergraduate medical education; Mexico

(I) Centro Médico Nacional Siglo XXI. Instituto Mexicano del Seguro Social. México, D.F.

(2) Hospital General de Zona. Medicina Familiar No. 2. Instituto Mexicano del Seguro Social, Saltillo, Coahuila, México

(3) Hospital General Regional No. I. Instituto Mexicano del Seguro Social, Acapulco, Guerrero, México

Fecha de recibido: 2 de junio de 2008 • Fecha de aprobado: II de noviembre de 2008

Solicitud de sobretiros: Francisco Raúl Sánchez Román. Centro Médico Nacional Siglo XXI, Instituto Mexicano del Seguro Social, Av. Cuauhtémoc 330 , Edificio C, Coordinación de Salud en el Trabajo, ler piso, col. Doctores. 06725 Delegación Cuauhtémoc, México, Distrito Federal, México. E-mail: raul.sanchezr@imss.gob.mx 
L os daños a la salud por accidentes y enfermedades de trabajo, además del incremento en la incidencia de cáncer y de las complicaciones por enfermedades crónico-degenerativas en personas de edad productiva, hoy en día representan un problema de salud pública que repercute en la economía y competitividad del país.

Durante 2007 fueron registrados, en el Instituto Mexicano del Seguro Social, 361244 accidentes de trabajo; 86167 accidentes de trayecto; 2691 enfermedades de trabajo; 17642 incapacidades permanentes; 1279 defunciones por riesgos de trabajo; 9970 demandas laborales y 14386 dictámenes de invalidez, que significaron un total de 11211385 días de incapacidad por riesgos de trabajo y 34056295 por enfermedad general. Lo anterior, en términos económicos, representó una erogación de 8148428060 de pesos por pago de incapacidades; 543133811 pesos por indemnizaciones globales y laudos y 1311958080 pesos por pensiones provisionales y temporales, ${ }^{1}$ consecuencias que en su mayor parte pudieron prevenirse.

A pesar de la importancia de la salud de los trabajadores y de su reconocimiento como eje fundamental para el desarrollo de las naciones, ${ }^{2}$ en nuestro país sigue siendo escasa la presencia de especialistas encargados de preservarla y mantenerla, como es el caso de la Medicina del Trabajo (MT), cuyos recursos humanos son insuficientes y su formación no ha sido acorde con el desarrollo nacional. ${ }^{3}$ A este respecto habría que considerar la influencia de las escuelas y facultades de medicina al ofrecer una formación que permita al egresado reconocer los alcances e implicaciones de la atención integral de este sector de la población, así como en la decisión de elegir determinada especialidad.

En las últimas décadas se ha señalado la incongruencia entre las necesidades sociales en salud y el quehacer de las instituciones encargadas de atenderla, así como entre la formación de recursos humanos, las necesidades sociales y los requerimientos de empresas e instituciones de salud respecto a los niveles de aptitud y orientación del personal que se contrata para cumplir con los objetivos planteados, con la idea de buscar la salud integral. ${ }^{4}$ En este sentido, la educación médica en México está conformada por un conjunto heterogéneo de instituciones con distintos grados de desarrollo y ofertas educativas, ya sean planteles públicos o privados, que poco han tomado en cuenta el "trabajo" como campo de estudio para la formación integral del médico general.

Se puede decir que la percepción habitual sobre el quehacer de la MT es pobre y con frecuencia se le considera una materia de poco interés cuando, de hecho, las empresas privadas representan una importante fuente de empleo para cientos de médicos, la mayoría sin nociones básicas en esta área especializada del conocimiento médico, lo que se traduce en falta de medidas preventivas específicas en los lugares de trabajo, diagnósticos erróneos o recaídas frecuentes, falta de vigilancia epidemiológica laboral, retrasos en la rehabilitación, despidos por falta de reincorporación laboral debido a secuelas permanentes y omisiones en el otorgamiento de indemnizaciones y pensiones por daños irreversibles a la salud, situaciones que reflejan deficiencias importantes de la política social.5,6

Lo anterior no es de extrañar si se toma en cuenta que la mayoría de los planes de estudio de escuelas y facultades de medicina tiene, como eje central la formación básica biomédica y clínica dirigida al tratamiento del individuo, mientras que el área sociomédica ocupa el menor porcentaje tanto de asignaturas como de horas de estudio. ${ }^{4}$

A pesar de los esfuerzos realizados por las principales asociaciones de facultades y escuelas de medicina, no se ha logrado un consenso satisfactorio para la formación de médicos que sean capaces de integrar los diversos aspectos que entraña el proceso salud-enfermedad de la población, situación importante dado el creciente interés de establecer estándares mundiales de formación. ${ }^{7}$

Con este trabajo se pretende esclarecer el estado actual que guarda la enseñanza de la MT en escuelas y facultades de medicina mexicanas, valorar algunas de sus características y proponer recomendaciones.

\section{Material y métodos}

Previa evaluación del comité de investigación, se llevó a cabo una encuesta descriptiva, de septiembre de 2005 a diciembre de 2006. Las facultades y escuelas de medicina fueron identificadas a través de la información disponible en internet por la Asociación Mexicana de Facultades y Escuelas de Medicina, la Asociación Nacional de Universidades e Instituciones de Educación Superior, la Comisión Interinstitucional para la Formación de Recursos Humanos para la Salud, el Instituto Internacional para la Educación Médica y el Directorio Internacional de Escuelas de Medicina de la Organización Mundial de la Salud. ${ }^{8-12}$ Una vez identificadas, se solicitó información por correo electrónico y por vía telefónica a los responsables de la gestión académica o de la asignatura; al no obtenerse respuesta por las vías anteriores, se buscó la información en las páginas web de las instituciones educativas. La información recabada se relacionó con la inclusión o no en el programa académico de la materia Medicina del trabajo o Salud en el trabajo y las siguientes características: si es o no obligatoria dentro del plan de estudios, departamento del que depende, grado de la carrera en que se imparte, 
créditos que otorga, duración, método de enseñanza, si se realizan prácticas en empresas, uso de nuevas tecnologías y cualidades de los profesores que imparten la materia. Para el análisis de la información, se utilizó estadística descriptiva y, cuando fue posible, se aplicó la prueba ji cuadrada para establecer diferencias entre las variables estudiadas.

\section{Resultados}

Se identificó un total de 75 escuelas y facultades de medicina distribuidas en 29 estados y el Distrito Federal. De ellas, $49(65 \%)$ son públicas y $26(35 \%)$ privadas (cuadro I). En $30(40 \%)$ se obtuvo respuesta por correo electrónico, en 32 (42\%) por vía telefónica, en 11 (15\%) a través de datos publicados en su página web y en dos (3\%) fue imposible obtener algún tipo de información. No todas las escuelas ofrecieron información completa debido a que al momento de contestar no contaban con datos suficientes y debido también al poco tiempo para detallar sus respuestas.

Del total, 39 (52\%) ofrecen la asignatura como obligatoria y $34(45 \%)$ no. La enseñanza de MT predomina de manera significativa $(p<0.02)$ en planteles públicos (cuadro II). De las universidades que no la ofrecen, algunas refirieron brindar temas relacionados, dentro de asignaturas como salud pública, sistema respiratorio, sistema osteomuscular, medicina preventiva o medicina social.

En los planteles que la ofrecen la asignatura depende de distintas áreas: en 10 (24\%) depende de salud pública; en ocho (20\%), de socio-medicina; en tres (7\%), de medicina legal; en dos (5\%), de ciencias clínicas; en uno $(2 \%)$, de senescencia; en uno (2\%), de ambiente y salud; en uno $(2 \%)$, de unidad médica; en uno ( $2 \%)$, de ciencias de la salud, y en $14(36 \%)$, no fue posible determinar su dependencia. La materia se ofrece entre el segundo y quinto año de la carrera; en un plantel (2\%), durante el segundo año; en siete (18\%), en tercer año; en 21 (55\%), en cuarto, y en 10 (25\%), en quinto año. En general no existe un criterio uniforme respecto a los créditos que le otorgan, ya que varían entre 0.5 y 300 , de acuerdo con la escuela y el plan de estudios. La duración de la materia es de un semestre, en 23 planteles $(66 \%)$ le dedican entre dos y tres horas por semana, con un rango de 1 a 8 horas (cuadro III); cabe hacer mención que, por lo menos en cuatro de ellas, la asignatura es compartida con otras materias como medicina legal, salud ambiental y salud del anciano, lo que reduce el tiempo de estudio. Acerca de las prácticas o visitas a empresas, 27 (69\%) refirieron contemplarlas como una actividad complementaria pero no obligatoria.
Cuadro I

EsCuelas Y FACULTADES DE MEDICINA IDENTIFICADAS POR ESTADO. MÉXICO, 2005

\begin{tabular}{|c|c|c|c|}
\hline Estado & Públicas & Privadas & Total \\
\hline Aguascalientes & 1 & 0 & 1 \\
\hline Baja California & 2 & 2 & 4 \\
\hline Campeche & I & 0 & 1 \\
\hline Chiapas & 1 & 2 & 3 \\
\hline Chihuahua & 2 & 0 & 2 \\
\hline Coahuila & 2 & 0 & 2 \\
\hline Colima & I & 0 & I \\
\hline Distrito Federal & 8 & 5 & 13 \\
\hline Durango & 2 & 0 & 2 \\
\hline Guanajuato & 1 & 1 & 2 \\
\hline Guerrero & 1 & 0 & 1 \\
\hline Hidalgo & I & 0 & I \\
\hline Jalisco & 3 & 2 & 5 \\
\hline Estado de México & 2 & 2 & 4 \\
\hline Michoacán & $\mathrm{I}$ & 0 & $\mathrm{I}$ \\
\hline Morelos & $\mathrm{I}$ & 0 & 1 \\
\hline Nayarit & $\mathrm{I}$ & 0 & I \\
\hline Nuevo León & 1 & 3 & 4 \\
\hline Oaxaca & $\mathrm{I}$ & $\mathrm{I}$ & 2 \\
\hline Puebla & $\mathrm{I}$ & $\mathrm{I}$ & 2 \\
\hline Querétaro & $\mathrm{I}$ & $\mathrm{I}$ & 2 \\
\hline San Luis Potosí & 1 & 0 & 1 \\
\hline Sinaloa & $\mathrm{I}$ & 0 & I \\
\hline Sonora & $\mathrm{I}$ & 0 & $\mathrm{I}$ \\
\hline Tabasco & $\mathrm{I}$ & 0 & 1 \\
\hline Tamaulipas & 2 & 4 & 6 \\
\hline Tlaxcala & 1 & 0 & 1 \\
\hline Veracruz & 5 & $\mathrm{I}$ & 6 \\
\hline Yucatán & $\mathrm{I}$ & I & 2 \\
\hline Zacatecas & $\mathrm{I}$ & 0 & 1 \\
\hline tal & 49 & 26 & 75 \\
\hline
\end{tabular}

Fuente: Directorios de escuelas y facultades de medicina

\section{Cuadro II}

EXISTENCIA DE LA MATERIA DE MEDICINA DEL TRABAJO EN UNIVERSIDADES PÚBLICAS Y PRIVADAS. MÉXICO, 2005-2006

\begin{tabular}{lccc}
$\begin{array}{c}\text { Inclusión de la } \\
\text { materia de MT }\end{array}$ & $\begin{array}{c}\text { Universidades } \\
\text { públicas (\%) }\end{array}$ & $\begin{array}{c}\text { Universidades } \\
\text { privadas (\%) }\end{array}$ & Total \\
Sí & $32(65)$ & $7(27)$ & $39(52)$ \\
\hline No & $15(31)$ & $19(73)$ & $34(45)$ \\
\hline No se determinó & $2(4)$ & 0 & $2(3)$ \\
\hline Total & $49(100)$ & $26(100)$ & $75(100)$
\end{tabular}

$\chi^{2} p<0.002$

Fuente: Encuesta en escuelas y facultades de medicina 
Cuadro III

Horas dedicadas a la semana a la materia DE MEDICINA DEL TRABAJO EN UNIVERSIDADES PÚBLICAS Y PRIVADAS. MÉXICO, 2005-2006

\begin{tabular}{cccc} 
Horas/semana & $\begin{array}{l}\text { Universidades } \\
\text { públicas (\%) }\end{array}$ & $\begin{array}{l}\text { Universidades } \\
\text { privadas (\%) }\end{array}$ & Total (\%) \\
\hline 1 & $\mathrm{I}(3)$ & $\mathrm{I}(17)$ & $2(6)$ \\
\hline 2 & $14(49)$ & 0 & $14(40)$ \\
\hline 3 & $7(24)$ & $2(32)$ & $9(26)$ \\
\hline 4 & $2(7)$ & $\mathrm{I}(17)$ & $3(8)$ \\
\hline 5 & $3(1 \mathrm{I})$ & $\mathrm{I}(17)$ & $4(\mathrm{II})$ \\
\hline 6 & $\mathrm{I}(3)$ & $\mathrm{I}(17)$ & $2(6)$ \\
\hline 8 & $\mathrm{I}(3)$ & 0 & $\mathrm{I}(3)$ \\
\hline Total & $29(100)$ & $6(100)$ & $35(100)$
\end{tabular}

$\chi^{2} p=0.318$

Fuente: Encuesta en escuelas y facultades de medicina
Sólo se obtuvo el plan de estudios de 15 universidades con la descripción de los temas de la asignatura, que evidenciaron gran desigualdad respecto a su número. Destacan como los más frecuentes: antecedentes históricos de la MT, legislación laboral, seguridad e higiene, prevención y atención de riesgos de trabajo (cuadro IV).

Como estrategia educativa predomina la clase magistral. El 95\% de los planteles que la imparten refirió contar con medios electrónicos en apoyo del proceso enseñanza-aprendizaje, aunque no se especificó de qué manera los utilizan.

Sobre el profesorado $15(38 \%)$ de las escuelas que ofrecen la asignatura refirió contar con especialistas en MT como profesores (cuadro V). Sólo la Universidad Nacional Autónoma de México cuenta con un programa de servicio social en empresas.

\section{Cuadro IV}

Temas abordados en la ASignatura de medicina del trabajo de ACUERDo Con los planes de estudio. MÉXICO 2005-2006

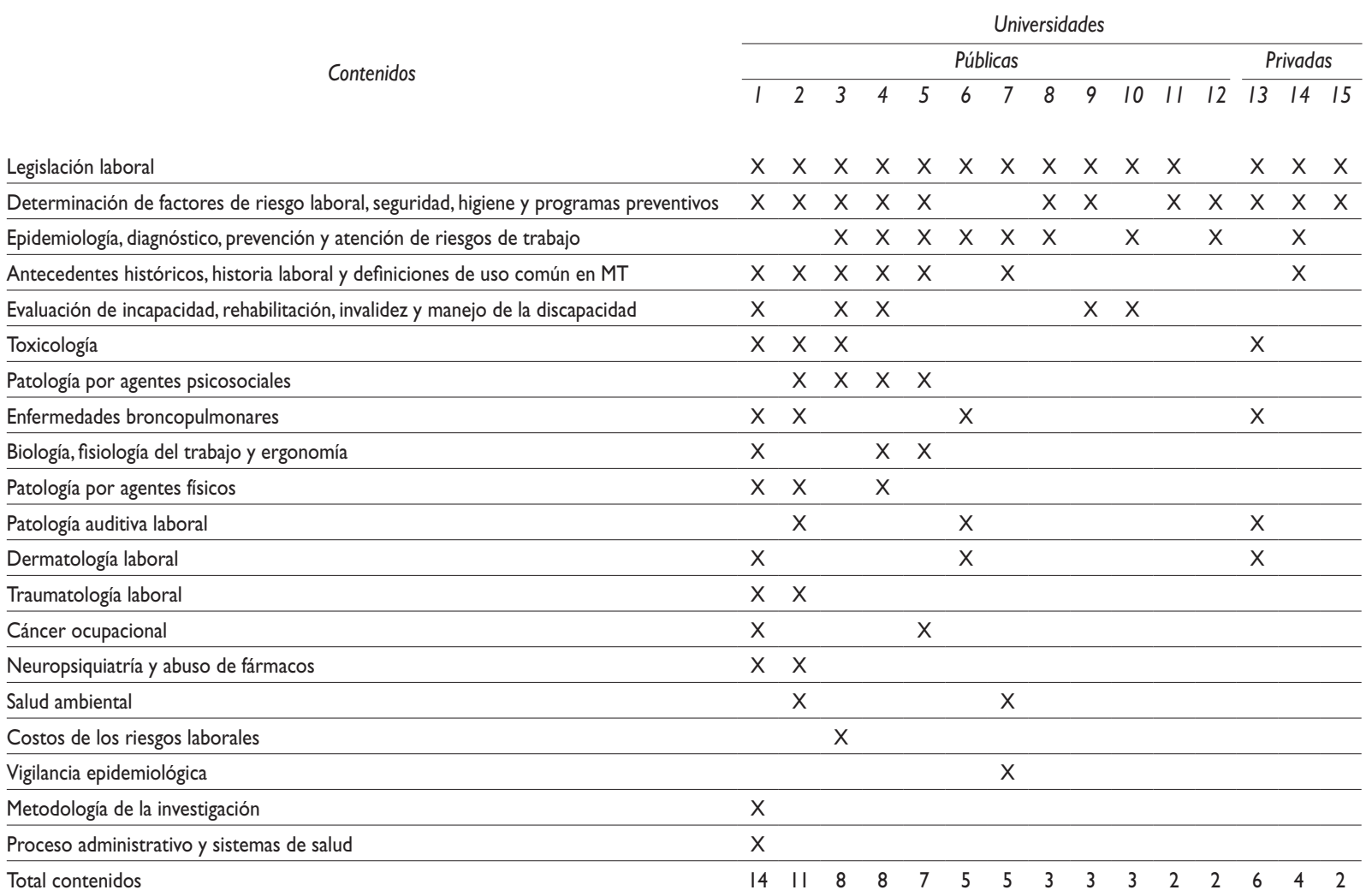

Fuente: Encuesta y plan de estudios de escuelas y facultades de medicina 


\section{CuadroV}

FORMACIÓN DEL PROFESOR QUE IMPARTE LA MATERIA DE MEDICINA DEL TRABAJO EN UNIVERSIDADES PÚBLICAS Y PRIVADAS. MÉXICO, 2005-2006

Especialistas (\%) No especialistas (\%) Total (\%)

\begin{tabular}{lccc} 
Universidades públicas & $12(80)$ & $13(87)$ & $25(83)$ \\
\hline Universidades privadas & $3(20)$ & $2(13)$ & $5(17)$ \\
\hline Total & $15(100)$ & $15(100)$ & $30(100)$
\end{tabular}

$\chi^{2} p=0.624$

Fuente: Encuesta en escuelas y facultades de medicina, México 2005-2006

\section{Discusión}

Este estudio proporciona evidencia sobre la limitada y heterogénea preparación que reciben los egresados de las escuelas y facultades de medicina respecto a MT ya que, por una parte, $45 \%$ no la contempla en sus currícula $y$, por otra, no existe un criterio uniforme en referencia a los créditos otorgados, duración y ubicación dentro del programa ni en los contenidos de la asignatura, a pesar de que desde 1935 se implementó por primera vez en el plan de estudios de la Facultad de Medicina de la Universidad Nacional Autónoma de México. ${ }^{13}$ Debido a los grandes avances en esta área durante las últimas décadas, en el ámbito internacional la MT ha sido un tópico que con el tiempo ha demostrado su importancia en la formación integral de los médicos y por lo mismo, aunque lento y con altibajos, su inclusión dentro de los currícula de pregrado ha ido en aumento. ${ }^{14-16}$

Llama la atención el hecho de que la enseñanza de la MT predomina significativamente en las escuelas públicas, lo que puede deberse a que intentan formar médicos generales en función de lo que las instituciones gubernamentales establecen como necesidades de atención médica de la población nacional, mientras que la mayor parte de las privadas orienta sus programas de formación hacia la especialización médica de sus egresados y llega erróneamente a considerar como elemento de calidad el hecho de ser o no seleccionado en el examen nacional de residencias médicas. ${ }^{17}$

La mayor parte de los planteles educativos proporciona la materia de MT durante el año previo al internado de pregrado, lo que coincide con la experiencia internacional; no así el tiempo que se le dedica, que en promedio es de tres horas menos en los planteles mexicanos. ${ }^{14-16}$

En países donde es más frecuente la enseñanza de MT, se observa un cambio positivo en la percepción de los estudiantes una vez que se aclara la relación entre trabajo y salud, el papel del especialista en MT y su interrelación con otras especialidades. ${ }^{18}$

Desde hace tiempo ha existido interés en cómo reformar los currícula de la carrera de medicina para integrar los conceptos básicos sobre los efectos nocivos del trabajo en la salud, ya que los conocimientos y actitudes de los estudiantes en relación con la materia han sido erráticos. ${ }^{19,20} \mathrm{~A}$ este respecto, el presente estudio evidencia una amplia gama de contenidos en los que no parece existir un propósito definido dentro del perfil de los egresados. Por otro lado, la manera de abordar los contenidos en la mayoría de los planteles es a través de clases magistrales que, aunado a la poca duración en horas por semana y a la falta de identidad de la MT en una área definida, influyen de manera negativa en la apreciación de la importancia de la materia.

Hay diversos métodos de enseñanza que han demostrado ser provechosos no sólo para el aprendizaje sino también para estimular el interés por la MT entre los que se encuentran la discusión de temas en pequeños grupos, discusión de casos clínicos, lectura crítica de la literatura, demostraciones de técnicas de evaluación en higiene y seguridad, búsquedas de información por medios electrónicos, visitas a empresas, visitas a centros de rehabilitación y de medicina del trabajo, ${ }^{21-25}$ situación que en nuestro medio es poco frecuente, ya que como estudiante y durante el internado de pregrado son raras las ocasiones en que se realiza alguna rotación por los servicios de MT.

Destaca el reducido número de especialistas que participan como docentes en la materia, factor que influye tanto en su implementación en los programas académicos como en el tiempo que se le dedica a su estudio. ${ }^{6} \mathrm{Al}$ respecto, en el año 2000 casi la tercera parte de los docentes de la carrera de medicina contaba al menos con licenciatura y nivel técnico superior, lo cual se puede considerar como un indicador de calidad en la educación médica. ${ }^{17}$

El servicio social en empresas, enfocado al desarrollo de actividades asistenciales y médico-preventivas supervisadas, ha documentado la reducción de hasta $30 \%$ en la incidencia de accidentes de trabajo. ${ }^{26}$

La MT, como tema de estudio para la formación integral de médico general, ha sido recomendada tanto por organismos internacionales ${ }^{27}$ como por estudios nacionales ${ }^{4}$ en la búsqueda de estándares que permitan al futuro médico integrarse a una sociedad cada vez más globalizada. Investigaciones sobre el seguimiento de los egresados podrían ayudar a conocer más sobre el empleo y desempeño laboral de los mismos, cuyos resultados deben ser un apoyo para la planeación de la educación médica. 
La disparidad en la enseñanza de aspectos básicos sobre MT en el pregrado explica, en parte, su poco desarrollo y su pobre reconocimiento social y profesional; además plantea serios problemas para la salud pública relacionados con la conservación de la salud que trastocan derechos y obligaciones de trabajadores y empleadores y por ende el avance económico del país. A pesar de esto, las instituciones educativas y de salud no han identificado esta problemática como un punto de interés suficiente para promover su estudio desde el pregrado de medicina, por lo que debe fortalecerse a través de:

- Su inclusión en los currícula de todas las escuelas y facultades de medicina

- Proporcionar durante un semestre un mínimo de ocho horas semanales en los ciclos académicos del último año de la licenciatura

- Abordar los siguientes temas de estudio: antecedentes de la medicina del trabajo, historia clínica laboral, legislación laboral, promoción de la salud en los lugares de trabajo, principios de seguridad e higiene industrial, identificación y evaluación de riesgos en el lugar de trabajo, vigilancia de la salud de los trabajadores, exámenes médicos laborales, evaluación de la capacidad para el trabajo, tiempos aproximados de recuperación por daños a la salud. Patología laboral: a) audiología: trauma acústico agudo y crónico; b) dermatología: dermatitis de contacto; c) neumología: neumoconiosis, bronquitis química y asma laboral; d) toxicología: intoxicación por disolventes orgánicos, plaguicidas y cancerígenos; e) osteomuscular: síndrome doloroso lumbar, cervical y trauma acumulativo

- Promover estrategias de aprendizaje que relacionen la teoría con la práctica, como la realización obligatoria de visitas a centros de trabajo y análisis de puestos de trabajo, así como actividades que desarrollen las aptitudes propias de la elaboración del conocimiento

- Impulsar la rotación por servicios de MT durante los ciclos académicos, el internado de pregrado y promover el servicio social en empresas

- Mejorar el perfil del personal académico contratado, lo cual es indispensable para elevar la calidad educativa.

En términos generales, los currícula de la carrera de medicina no han modificado los esquemas formativos implantados desde hace varias décadas y en los que poco se ha tomado en cuenta a la MT como parte complementaria de su formación debido a la falta de un diagnóstico que oriente su planeación y adaptación a los tiempos y necesidades de la sociedad.

La enseñanza de la MT en el pregrado carece de una definición clara, con una estructuración de contenidos inconexos y sin un propósito concreto dentro del perfil de los egresados. En este sentido, su enseñanza debería estar dirigida a elementos teórico-prácticos para que el futuro médico general pueda atender de forma idónea a su comunidad, cualquiera que sea su campo de acción, incluido el trabajo en empresas públicas o privadas, el cual suelen enfrentar en el terreno profesional con un importante déficit de conocimientos en la materia.

Lo anterior resulta trascendente ya que, por una parte, una gran proporción de egresados no obtendrá un sitio para cursar una especialidad médica debido a la reducida oferta de plazas, por lo que deberá practicar la medicina general en diversos escenarios, desde el consultorio privado en el que seguramente atenderá a trabajadores, hasta su ejercicio en una empresa en la que pueda ayudar no a tratar a los accidentados y enfermos, sino en la prevención de accidentes y enfermedades y así evitar el deterioro del trabajador. También una gran proporción de egresados ignora la existencia y los alcances de la especialidad como opción de desarrollo, pues además del componente sociomédico comprende importantes elementos clínicos y biomédicos necesarios para la correcta atención de este sector tan importante de la población.

\section{Agradecimientos}

A las facultades y escuelas de medicina que desinteresadamente participaron en este estudio. A la señorita Judith Juárez, por su participación en la realización y el seguimiento de la encuesta.

\section{Referencias}

I. Instituto Mexicano del Seguro Social. Sistema de información sobre las prestaciones económicas. Información estadística sobre el gasto total de las prestaciones económicas 2007.

2. Organización Panamericana de la Salud / Organización Mundial de la Salud. Salud de los trabajadores en la región de las Américas. $41^{\circ}$ Consejo Directivo. 1999.

3. Sánchez RF, Pérez PA, Sánchez VP, Ortega EM, Pérez MP, Haro GL. Reflexiones en torno a los 40 años de la Medicina del Trabajo en México. Rev Med Inst Mex Seguro Soc 2007;45(4):403-4I2.

4. Fernández PJ. Las carreras de medicina en México. Perfiles Educativos 1996;73(18).

5. Sánchez RF, Juárez PC,Aguilar MG, Haro GL, Borja AV, Claudio L. Occupational Health in Mexico. Int J Occup Environ Health 2006; 12:346-354. 
6. Wynn PA, Williams NR, Snashall D, Aw TC. Undergraduate occupational health teaching in medical schools. Not enough of a good thing? Occup Med 2003;53:347-348.

7. Knight J. El nuevo mundo de la movilidad académica: programas y proveedores transfronterizos. Perfiles Educativos 2006;28: I I-54.

8. Asociación Mexicana de Facultades y Escuelas de Medicina [consultado 2005 sept 20]. Disponible en: http://www.amfem.edu.mx

9. Asociación Nacional de Universidades e Instituciones de Educación Superior [consultado 2005 sept 2I]. Disponible en: http://www.anuies. $\mathrm{mx} / \mathrm{la}$ anuies/diries/

10. Comisión Interinstitucional para la Formación de Recursos Humanos para la Salud. Médicos Mexicanos Inscritos, Sustentantes, Seleccionados y Resultados por Facultad y Escuela de Medicina [consultado 2005 sept 22]. Disponible en: http://www.cifrhs.org.mx

II. Instituto Internacional para la Educación Médica [consultado 2005 oct 14]. Disponible en: http://www.iime.org/database/northam/mexico.htm 12. Organización Mundial de la Salud, directorio internacional de escuelas de medicina [consultado 2005 nov 4]. Disponible en: http://whqlibdoc. who.int/publications/WDMS/WDMS_J-P.pdf

13. Roldán VU. La enseñanza de la medicina del trabajo en México. Iniciación, desarrollo y proyecciones futuras. Gac Med Mex 1965;95(8):767-772.

14. Burstein JM, Levy BS. The teaching of occupational health in US medical schools: little improvement in 9 years. Am J Public Health 1994;84(5):846-849.

15. Shanahan EM, Murray AM, Lillington T, Farmer EA. The teaching of occupational and environmental medicine to medical students in Australia and New Zealand. Occup Med 2000;50(4):246-250

16. Wynn PA, Aw TC, Williams NR, Harrington M. Teaching of occupational medicine to undergraduates in UK schools of medicine. Med Educ 2002;36:697-70I
17. De la Garza AJ. Reflexiones sobre la calidad de la carrera de medicina en México. Gac Med Méx 2005; 14 I (2): 129- 4 II.

18. Franco G. Students perception of occupational medicine.J Occup

Environ Med 1996;38(3):240-24I.

19. Lee WR. Training for occupational medicine. Postgrad Med J

1989;65:156-160.

20. Sokas RK, Cloeren M. Occupational health and clinical training. J Occup Med 1987;29(5):414-4I6.

2I. Koh D, Chia KS, Jeyaratnam J, Chia SE, Singh J. Case studies in occupational medicine for medial undergraduate training. Occup Med 1995;45:27-30

22. Fletcher G,Agius RM. The special study module: a novel approach to undergraduate teaching in occupational medicine. Occup Med 1995;45(6):326-328.

23. Grime P, Williams S, Nicholson S.A new undergraduate teaching session in occupational medicine. Med Educ 2003;37:1033-1034.

24. Newson-Smith MS, Nicol AG. Teaching of occupational medicine to undergraduates. Occup Med 2004:54(2): I34-135.

25. Grime P, Williams S, Nicholson S. Medical students' evaluation of a teaching session in occupational medicine: the value of a workplace visit. Occup Med 2006;56: I I0-I I4.

26. González CE. Programa de servicio social en medicina del trabajo. Condiciones de trabajo. Secretaría del Trabajo y Previsión Social. 1978;3(2): 67-72

27. Schwarz MR, Wojtczak A. Institute for International Medical Education. Global minimum essential requirements in medical education. Med Teach 2002;24(2):125-129. 